Ricardo Halpern 1 Fernando C. Barros 2 Bernardo L. Horta 2 Cesar G. Victora 2

\section{Desenvolvimento neuropsicomotor aos 12 meses de idade em uma coorte de base populacional no Sul do Brasil: diferenciais conforme peso ao nascer e renda familiar}

\author{
Developmental status at 12 months of age \\ in a cohort of children in southern Brazil: \\ differences according birthweight and family \\ income
}

1 Departamento Materno-Infantil, Faculdade de Medicina, Universidade Federal de Pelotas. C. P. 464, Pelotas, RS 96001-970, Brasil.

2 Departamento de Medicina Social, Faculdade de Medicina, Universidade Federal de Pelotas. C. P. 464, Pelotas, RS 96001-970, Brasil

\begin{abstract}
This study evaluated all children born in 1993 in hospitals from Pelotas, Rio Grande do Sul, Brazil. Besides a perinatal study, a sample of $20 \%$ (1400 children) was followed through home visits during the first year. During these visits the nutritional status was assessed and a screening test for development (Denver II Test) was performed. Thirty-four per cent of the children assessed at 12 months failed this screening. Failure was associated with socioeconomic status, with low-income children presenting twice the risk of those from more affluent families $(p<0,001)$. Failure in the screening test was also associated with birthweight, and children weighing less than 2,500 $\mathrm{g}$ showed three times the risk of those with a birthweight equal to or greater than $2,500 \mathrm{~g}(p<0,001)$. In addition, children weighing less than $2000 \mathrm{~g}$ at birth were at three times the risk of failing the test as compared to those weighing between $2000 \mathrm{~g}$ and 2499g. Results suggest that birthweight and family income are strongly related to the potential risk of developmental delays at the age of 12 months. It also points to the need for systematic developmental screening and early intervention programs in children at risk.
\end{abstract}

Key words Child Development; Screening; Low Birthweight; Early Intervention; Denver II

Resumo Os 5.304 nascimentos hospitalares ocorridos em 1993 em Pelotas, Rio Grande do Sul, foram investigados. Além da avaliação perinatal de todos os recém-nascidos, 20\% (1.400 crianças) foram acompanhadas, por meio de visitas domiciliares, durante o primeiro ano. Nestas visitas, era realizado o Teste de Denver II para avaliação do desenvolvimento. Aos 12 meses, das 1.362 crianças avaliadas, 463 (34\%) apresentaram teste sugestivo de atraso no desenvolvimento. Este resultado esteve associado com a renda familiar, tendo sido duas vezes mais frequente entre as crianças de famílias mais pobres do que entre as de melhor situação sócio-econômica $(p<0,001)$. Falha no teste também esteve inversamente associada com o peso de nascimento, com as crianças de baixo peso apresentando um risco três vezes maior do que aquelas com peso ao nascer igual ou superior a $2.500 \mathrm{~g}(p<0,001)$. Além disso, crianças que nasceram com peso inferior a $2.000 \mathrm{~g}$ apresentaram um risco três vezes maior do que aquelas com peso entre $2.000 \mathrm{~g} e$ 2.499g. Os resultados indicam que o peso ao nascer e a situação sócio-econômica estão fortemente associados com potenciais atrasos no desenvolvimento de crianças aos 12 meses de idade. Apontam, também, para a necessidade de triagem sistemática do desenvolvimento e programas de intervenção precoce em grupos de risco.

Palavras-chave Desenvolvimento Infantil; Baixo Peso ao Nascer; Triagem; Teste de Denver II 


\section{Introdução}

Crianças vivendo em países em desenvolvimento enfrentam um duplo desafio: estão mais sujeitas a nascerem com baixo peso, seja por parto pré-termo, e/ou retardo de crescimento intra-uterino, sofrendo os problemas perinatais decorrentes (Victora et al., 1989; Stanley \& Alberman, 1984) e freqüentemente vivem em ambientes familiares desfavoráveis, onde a estimulação e o suporte social são inadequados. Esta seqüência de eventos aumenta o risco de atraso em seu desenvolvimento cognitivo, físico e social (Brooks Gunn, 1990; Werner et al., 1967).

Apesar da maioria dos estudos sobre desenvolvimento serem realizados em populações selecionadas de países desenvolvidos (Saigal et al.,1991; Vohr, 1991), pesquisas realizadas em países do terceiro mundo têm mostrado importante associação entre o desenvolvimento neuropsicomotor da criança, seu estado nutricional e a situação sócio-econômica da família (Grantham McGregor et al., 1982). Mesmo com as evidências da importância de programas de intervenção precoce (Simeonsson et al., 1982) e o sucesso dessas intervenções em crianças de risco em países em desenvolvimento (Grantham McGregor et al., 1987), poucos avanços têm ocorrido nesta área. Possíveis explicações para esta lacuna são a complexidade das razões de atraso, a inexistência de sistemas de vigilância, o que dificulta a identificação e acompanhamento das crianças vulneráveis, e a não utilização de instrumentos adequados para triagem de crianças com risco de atraso no desenvolvimento neuropsicomotor (Torburn, 1990).

Os objetivos do presente estudo foram identificar crianças em risco para atrasos no desenvolvimento neuropsicomotor em uma coorte com 12 meses de idade e investigar a possível associação de um potencial atraso com o peso ao nascer e a situação sócio-econômica.

\section{Metodologia}

Em 1993, todas as mães das 5.304 crianças nascidas nos hospitais de Pelotas, que residiam na zona urbana da cidade, foram entrevistadas após o parto. As informações coletadas através de questionário diziam respeito a variáveis reprodutivas, demográficas, sócio-econômicas e ambientais (Victora et al., 1996). Foi realizada antropometria materna e do recém-nascido, sendo também calculada a idade gestacional pela data da última menstruação e por meio do método de Dubowitz (Dubowitz et al., 1970). Uma amostra aleatória de $20 \%$ das crianças foi visitada em casa aos 12 meses de idade.

O desenvolvimento neuropsicomotor destas crianças foi testado através de uma nova versão do Teste de Triagem de Denver (Frankenburg et al., 1990), adaptado para o Português, que foi delineado para ser utilizado em crianças desde o nascimento até a idade de seis anos. Este teste foi escolhido por ser o mais utilizado por profissionais da área da saúde para triagem em populações assintomáticas (Meisels, 1989), além de ser de treinamento fácil e administração rápida (20 min). O teste consiste em 125 itens que são divididos em quatro grupos: a) Pessoal-Social: aspectos da socialização da criança dentro e fora do ambiente familiar; b) Motricidade fina: coordenação olhomão, manipulação de pequenos objetos; c) Linguagem: produção de som, capacidade de reconhecer, entender e usar linguagem e d) Motricidade ampla: controle motor corporal, sentar, caminhar, pular e todos os demais movimentos realizados pela musculatura ampla. Estes itens são administrados diretamente à criança e em alguns deles é solicitado que a mãe informe se a criança realiza ou não determinada tarefa.

Os entrevistadores foram previamente treinados na administração do teste, através de metodologia estabelecida no manual de treinamento do teste original (Frankenburg et al., 1990), e não foram informados das hipóteses da investigação. O material utilizado para administração do teste foi reproduzido a partir do kit original que acompanhava o manual de instruções. As respostas eram codificadas em passa, falha, ou recusa (não testável), de acordo com a habilidade da criança em realizar determinado item. Em alguns itens pré-determinados a informação da mãe servia como resposta. Segundo a norma do teste (Frankenburg et al., 1990), foram considerados casos indicativos de atraso aqueles em que a criança falhou em dois ou mais itens do teste, não importando a área em que a falha ocorreu. Da mesma maneira que em outras etapas dos acompanhamentos (Victora et al., 1996), uma amostra de $5 \%$ dos casos foi revisitada, com a finalidade de verificar a veracidade da informação e os critérios utilizados na pontuação dos itens.

Como a amostra continha crianças nascidas pré-termo, a idade deste grupo foi ajustada, subtraindo-se da mesma o número de semanas que a criança havia nascido antes de completar 37 semanas de gestação. Desta forma, a avaliação foi feita conforme a idade de desenvolvimento, impedindo uma superesti- 
mativa das crianças que apresentariam o Teste de Denver II com alterações pelo fato de terem nascido pré-termo.

Os dados foram preparados no programa Epiinfo 6,0 (Dean et al., 1990) e a análise foi realizada no programa SPSS versão 6.1 (Norussis, 1994). Na análise bivariada foi utilizado o teste do qui-quadrado para tabelas de contingência, com nível de significância de 5\%, e a análise multivariada foi realizada através de regressão logística, onde foram ajustadas as possíveis variáveis de confusão.

\section{Resultados}

Das 1.460 crianças sorteadas para participarem do estudo, 1.362 (94,7\%) foram efetivamente acompanhadas. Houve 11 recusas e 66 crianças não foram localizadas pelo endereço. Do total das crianças visitadas, 463 (34\%) apresentaram teste indicativo de atraso no desenvolvimento, com falha em dois ou mais itens do teste. Houve diferenças importantes quanto ao setor do desenvolvimento comprometido: a escala de motricidade ampla mostrou o maior percentual de atraso $(14,9 \%)$, seguida da escala pessoal-social (5,5\% das crianças) e, em proporção bem menor, da área de motricidade fina (1,6\%).

Devido ao número pequeno de casos por grupos específicos, os resultados serão descritos considerando o resultado global do teste. $\mathrm{O}$ percentual de atraso de acordo com o gênero da criança não mostrou diferença significativa, com os meninos apresentando um percentual de atraso de $35 \%$ e as meninas de $33 \%$.

A Tabela 1 mostra a proporção de atraso de acordo com a distribuição de renda familiar. Nota-se que o percentual de atraso diminui conforme aumenta a renda da família, mostrando uma tendência linear estatisticamente significativa, com as crianças mais pobres apresentando duas vezes mais falha no teste do que as pertencentes a famílias de maior renda (razão de odds=2,04; IC 95\% 1,36-3,05).

$O$ peso do nascimento esteve significativamente associado com o desempenho neuropsicomotor das crianças. O percentual de indicativo de atraso aumentou nos grupos de menor peso de nascimento (Figura 1).

Como existe uma forte relação entre peso de nascimento e renda familiar, a associação entre peso ao nascer e resultados negativos no Teste de Denver foi analisada controlando o possível efeito de confusão da renda familiar. A Tabela 2 mostra, que mesmo após este ajuste, as diferenças entre os grupos de peso permaneceram significativas. As crianças que nasce-
Tabela 1

Indicativo de atraso no desenvolvimento com um ano de idade, por grupos de renda, segundo Teste de Denver II. Pelotas, 1993.

\begin{tabular}{|c|c|c|c|c|c|}
\hline & \multicolumn{2}{|c|}{ Sem atraso } & \multicolumn{2}{|c|}{ Suspeito ( $\geq$ itens)* } & \multirow[t]{2}{*}{$\mathbf{n}$} \\
\hline & $\mathrm{n}$ & $\%$ & $n$ & $\%$ & \\
\hline$\leq 1$ s. m. & 144 & 58,9 & 100 & 41,1 & 244 \\
\hline $1,1-3$ s. m. & 393 & 64,2 & 219 & 35,8 & 615 \\
\hline $3,1-6$ s. m. & 208 & 69,5 & 91 & 30,5 & 299 \\
\hline$>6$ s. m. & 155 & 74,4 & 53 & 25,5 & 208 \\
\hline
\end{tabular}

* $\mathrm{p}<0,002$

s. m.: salário mínimo

Tabela 2

Razão de Odds para indicativo de atraso no desenvolvimento com um ano de idade conforme peso ao nascer. Pelotas, RS.

\begin{tabular}{lrrccc}
\hline \multirow{2}{*}{ Grupos de peso } & \multicolumn{2}{c}{ Atraso } & RO (bruto) & RO (ajustado)* & IC 95\% \\
& \multicolumn{1}{c}{$\mathrm{n}$} & $\%$ & & & \\
\hline até $1.999 \mathrm{~g}$ & 23 & 73,2 & 14,4 & 15,0 & $(5,32-42,75)$ \\
$2.000-2.499 \mathrm{~g}$ & 106 & 50,0 & 4,90 & 4,89 & $(2,45-9,75)$ \\
$2.500-2.999 \mathrm{~g}$ & 325 & 41,7 & 3,51 & 3,46 & $(1,87-6,41)$ \\
$3.000-3.499 \mathrm{~g}$ & 559 & 31,1 & 2,24 & 2,27 & $(1,24-4,13)$ \\
$3.500-3.999 \mathrm{~g}$ & 262 & 24,9 & 1,62 & 1,63 & $(0,52-3,10)$ \\
$\geq 4.000 \mathrm{~g}$ & 83 & 16,9 & 1,00 & 1,00 & \\
\hline
\end{tabular}

* ajustado para renda familiar

ram com baixo peso (menos de $2.500 \mathrm{~g}$ ) apresentaram um risco três vezes maior de atrasarem o seu desenvolvimento em relação àquelas que nasceram com peso igual ou superior a $2.500 \mathrm{~g}(\mathrm{p}<0,001)$. Da mesma forma, as crianças que nasceram com peso inferior a $2.000 \mathrm{~g}$ apresentaram um risco três vezes maior do que aquelas com peso entre $2.000 \mathrm{~g}$ e $2.499 \mathrm{~g}$ ( $\mathrm{p}<$ 0,001).

Da mesma forma que a renda poderia exercer efeito sobre o peso de nascimento, foi controlado o possível efeito do peso ao nascer na associação entre atraso no desenvolvimento e renda familiar (Tabela 3). Não houve modificação no risco após este controle, o que sugere que tanto o peso ao nascer quanto a renda familiar possuem efeitos independentes sobre o desenvolvimento neuropsicomotor.

\section{Discussão}

Os riscos para atrasos no desenvolvimento estão concentrados em três grupos principais: aqueles com diagnóstico estabelecido, como, 
por exemplo, os erros inatos do metabolismo; o grupo de crianças com risco biológico geralmente relacionado às condições de gestação e nascimento; e aqueles com risco ambiental, onde estão concentradas as dificuldades relacionadas com uma estrutura familiar deficiente, características sócio-econômicas desfavoráveis e cuidados de saúde inadequados (Tjossem, 1976; Guralnick \& Bennett, 1987). Estes grupos não são excludentes, definindo a característica multifatorial dos atrasos do desenvolvimento infantil. Estudos recentes demonstraram que o efeito cumulativo de fatores de risco múltiplos aumenta a probabilidade do desenvolvimento da criança ser comprometido (Meisels \& Wasik, 1990; Sameroff et al., 1987). Desta forma, as crianças de países subdesenvolvidos concentram a grande maioria das possíveis causas que levam a um atraso do desenvolvimento neuropsicomotor.

Tabela 3

Razão de Odds para indicativo de atraso no desenvolvimento com um ano de idade conforme renda familiar. Pelotas, RS.

\begin{tabular}{|c|c|c|c|c|c|}
\hline \multirow[t]{2}{*}{ Renda familiar } & \multicolumn{2}{|c|}{ Atraso } & \multirow[t]{2}{*}{ RO (bruto) } & \multirow{2}{*}{ RO (ajustado)* } & \multirow[t]{2}{*}{ IC $95 \%$} \\
\hline & $\mathrm{n}$ & $\%$ & & & \\
\hline$\leq 1$ s. m. & 244 & 41,2 & 2,04 & 1,90 & $(1,26-2,88)$ \\
\hline $1,1-3$ s. m. & 615 & 36,0 & 1,62 & 1,57 & $(1,09-2,25)$ \\
\hline $3,1-6$ s. m. & 299 & 30,7 & 1,28 & 1,21 & $(0,80-1,82)$ \\
\hline$>6$ s.m. & 208 & 26,0 & 1,00 & 1,00 & \\
\hline
\end{tabular}

* ajustado para peso ao nascer
O presente estudo revela a magnitude do efeito do peso ao nascer e da situação sócioeconômica sobre o desenvolvimento infantil aos 12 meses de idade e é compatível com pesquisas realizadas em outros locais (McCormick, 1989; Brooks Gunn, 1990; Vohr, 1991). Embora as crianças de famílias de baixa renda tenham apresentado um risco duas vezes maior de falharem no teste de Denver II, o peso ao nascer mostrou uma associação ainda mais forte com indicativos de atrasos, sendo responsável pela maior parte da variação encontrada. A análise por regressão logística realizada demonstrou que os efeitos de peso ao nascer e renda são independentes e confirmou a importância de ambos na determinação do retardo do desenvolvimento infantil. É óbvio que outras variáveis, como a educação materna, o tamanho da família e a ocupação do pai, que neste estudo não foram abordadas, são também importantes preditores do desenvolvimento futuro da criança (Broman, 1984).

Estes resultados contrastam, parcialmente, com a avaliação realizada em uma outra coorte de crianças de Pelotas (Victora et al., 1989). Neste estudo, o efeito da renda foi mais importante do que o do peso ao nascer como determinante de atraso. Uma possível explicação para esta variação é o diferente instrumento utilizado, o Teste de Griffits (Griffits, 1970), que tem finalidade diagnóstica e não de triagem, como o teste de Denver. Entretanto, talvez a principal razão para a diferença entre estes estudos é que no da coorte de 1982 as crianças tinham idade bem superior - média de 53 meses, e se

Figura 1

Falha no teste de Denver II aos 12 meses e peso ao nascer.

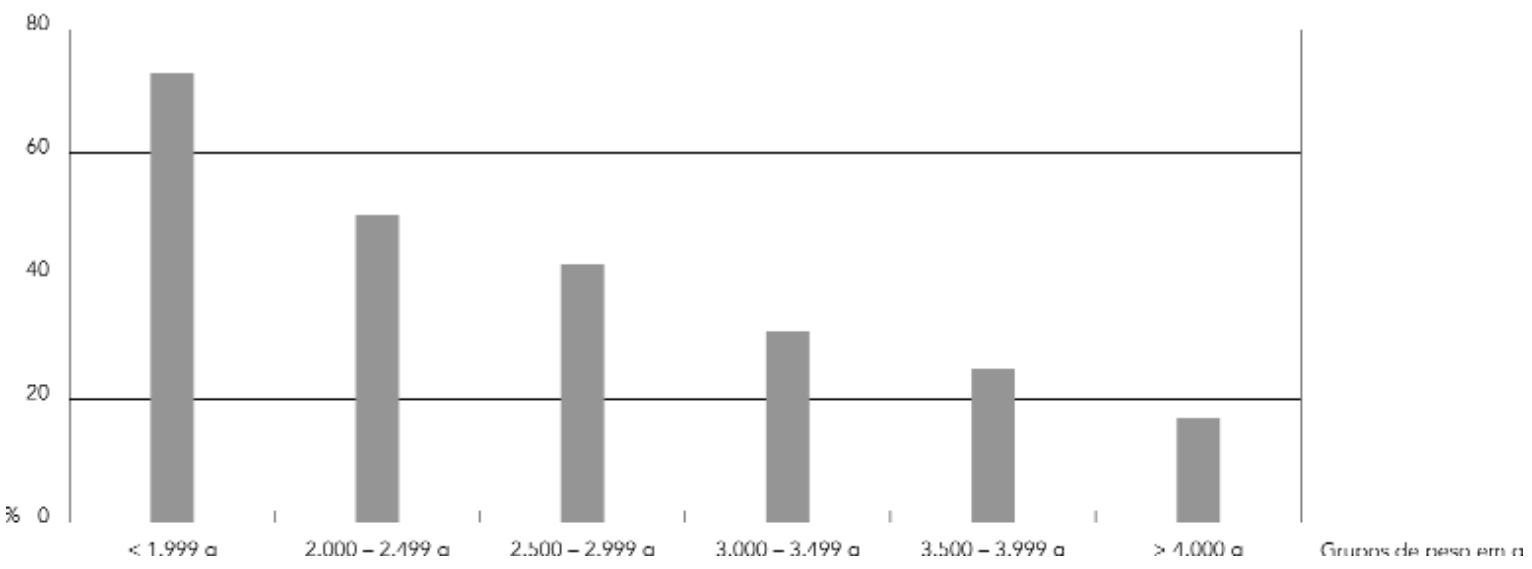


poderia esperar que, com o tempo, as variáveis relacionadas com estrutura familiar e fatores sócio-econômicos adquirissem maior importância, superando o efeito do peso ao nascer (Meisels \& Wasik, 1990).

Estudos com as versões anteriores do teste de Denver apontam para algumas falhas na normatização do instrumento, entre outras o fato de apresentar uma sensibilidade baixa (Meisels, 1989). A nova versão do instrumento apresenta uma normatização e validação cuidadosa, e os aspectos apontados como limitações foram corrigidos, sendo detalhadamente discutidos nas normas do teste (Frankenburg et al., 1990). Mesmo com o cuidado na elaboração desta nova versão, devemos considerar que diferenças culturais poderiam afetar o resultado obtido, como já foi mostrado em estudos anteriores (Sriyaporn et al., 1994; Lim et al., 1994). Apesar destas possíveis limitações, a inexistência de instrumento padronizado para o Português, associada à necessidade de uma identificação de crianças de risco, justifica o uso do Teste de Denver II.

A elevada proporção de crianças com Teste de Denver II com resultado negativo deve ser interpretada com a devida cautela. Em primeiro lugar, cabe lembrar que testes de triagem alertam para um risco potencial que pode ser confirmado ou não através de um acompanhamento sistemático e mediante testes diagnósticos. Além disso, a avaliação do desenvolvimento em um único momento não permite que se determine de forma definitiva um atraso no desenvolvimento da criança. De qualquer forma, o estudo sugere que crianças sujeitas a potenciais atrasos de desenvolvimento podem ser identificadas já ao nascimento, por meio do conhecimento do peso ao nascer e da situação sócio-econômica da família. Através desta identificação precoce pode ser possível o estabelecimento de programas de intervenção que visem à prevenção de distúrbios do desenvolvimento.

\section{Referências}

BROMAN, S., 1984. The collaborative perinatal project: an overview. In: Handbook of Longitudinal Research. Vol 1. Birth and Childhood Cohorts (S. Mednick, M. Harway \& K. Finelle, eds.), pp. 185215, New York: Praeger.

BROOKS GUNN, J., 1990. Enhancing the development of young children. Current Opinion in Pediatrics, 2:873-877.

DUBOWITZ, L. M. S.; DUBOWITZ, V. \& GOLDBERG, C., 1970. Clinical assessment of gestational age in newborn infant. The Journal of Pediatrics 1:77.

DEAN, A. G.; DEAN J. A.; BURTON A. H.; DICKER R. C. , 1990. Epi Info, Version 5.01: a Word Processing Database and Statistics Program for Epidemiology on Microcomputers. Atlanta: Center for Disease Control and Prevention.

FRANKENBURG, K. W.; DODDS, J.; ARCHER, P.; BRESNICK, B.; et al., 1990. Denver II: Technical Manual and Training Manual. Denver: Denver Developmental Materials Inc.

GURALNICK, M. J. \& BENNETT, F. C., 1987. A framework for early intervention. In. The Effectiveness of Early Intervention for At-Risk and Handicapped Children (M. J. Guralnick \& F.C. Bennett, eds.), pp. 3-32, Orlando: Academic Press.

GRANTHAM McGREGOR, S. M.; POWEL, C.; STEWART, M. \& SCHOFIELD, W. N., 1982. Longitudinal study of growth and development of young Jamaican children recovering from severe proteinenergy malnutrition. Developmental Medicine Child Neurology, 24:321-331.
GRANTHAM McGREGOR, S. M.; SCHOFIELD, W. N. \& POWEL, C., 1987. Development of severely malnourished children who received psychosocial stimulation: six-year follow-up. Pediatrics, 79: 247-254.

GRIFFITS, R., 1970. The Abilities of Young Children: A Comprehensive System of Mental Measurement for the First Eight Years of Life. London: Child Development Research.

LIM, H. C.; CHAN, T. \& YOONG, T., 1994. Standardization and adaptation on the Denver Developmental Screening Test (DDST) and Denver II for use in Singapore children. Singapore Medical Journal, 35:156-160.

McCORMICK, M. C., 1989. Long-term follow-up of infants discharged from neonatal intensive care units. Journal of American Medical Association, 261:1767-1772.

MEISELS, J. S., 1989. Can developmental screening test identify children who are developmentally at risk? Pediatrics, 83:578-585.

MEISELS, J. S. \& WASIK, B. A., 1990. Who should be served? Identifying children in need of early intervention In: Handbook of Early Intervention (S. J. Meisels \& J. Shonkoff, eds.), pp. 605-632, Cambridge: Cambridge University Press.

NORUSSIS, M. J., 1994. SPSS/PC 6.1. Statistical Package for Social Science. Chicago: SPSS Inc.

SAMEROFF, A.; SEIFER, R.; BAROCAS, R.; ZAX, M. \& GREENSPAN, S., 1987. Inteligence quocient scores of 4 years old children: social emotional risk factors. Pediatrics, 79:343-350. 
SAIGAL, S.; SZATMANI, P.; ROSEMBAUM, P.; CAMPBELL, D.; KING S., 1991. Cognitive abilities and school performance of extremely low birth weight children and matched term control children at age 8 years: a regional study. Journal of Pediatrics, 118:751-760.

SIMEONSSON, R. J.; COOPER, D. H. \& SCHEINER, A. P., 1982. A review and analysis of the effectiveness of early intervention programs. Pediatrics, 69: 635-641.

STANLEY, F. \& ALBERMAN, E., 1984. Birthweight, gestational age and cerebral palsies. In: The Epidemiology of the Cerebral Palsy (F. Stanley \& E. Alberman, eds.), pp. 57-68, Oxford: Clinics in Developmental Medicine no 87. Spastics International Medical Publications, Blackwell Scientific Publication Ldt.

SRIYAPORN, P. P.; PISSASOONTORN, W. \& SAKDISAWADI, O., 1994. Denver Developmental Screening Test survey of Bangkok children. Asian Pacific. Journal of Public Health, 7:173-177.

TJOSSEM, T. D., 1976. Intervention Strategies for High Risk Infants and Young Children. Baltimore: University Park Press.
TORBURN, J. M., 1990. Childhood disability in developing countries: basic issues. In: Practical Approaches to Childhood Disability in Developing Countries: Insights from Experience and Research (J. M. Thorburn \& J. Marfo, eds.), pp. 3-28, St John's: Project Seredec Memorial University of Newfoundland.

VICTORA, C. G.; BARROS, F. C. \& VAUGHAN, J. P., 1989. Epidemiologia da Desigualdade. 2a ed., São Paulo: Hucitec.

VICTORA, C. G.; BARROS, F. C.; HALPERN, R. et al., 1996. Estudo longitudinal da população materno-infantil da região urbana do Sul do Brasil, 1993: aspectos metodológicos e resultados preliminares. Revista de Saúde Pública, 30:34-45.

VOHR, R. B., 1991. Preterm cognitive development: biologic and environmental influences. Infant and Young Children, 3:20-29.

WERNER, E.; SIMONIAN, K.; BIERMAN, J.; et al., 1967. Cumulative effect of perinatal complications and deprived social environment on physical, intellectual and social development of preschool children. Pediatrics, 39:480-505. 